\title{
Desquamative Interstitial Pneumonia Complicated with IgG4-related Lung Disease
}

\author{
Hideaki Yamakawa ${ }^{1,2}$, Yoshihiro Suido ${ }^{1}$, Shinko Sadoyama ${ }^{1}$, Yumie Yamanaka ${ }^{1,2}$, \\ Satoshi Ikeda ${ }^{1}$, Hideya Kitamura ${ }^{1,2}$, Tomohisa Baba ${ }^{1}$, Koji Okudela ${ }^{3}$, \\ Tamiko Takemura ${ }^{4}$ and Takashi Ogura ${ }^{1}$
}

\begin{abstract}
As an idiopathic interstitial pneumonia, desquamative interstitial pneumonia (DIP) is an uncommon form of interstitial lung disease and is considered to be a smoking- or dust inhalation-related interstitial pneumonia in the majority of cases. However, the details regarding immunoglobulin G4 (IgG4)-related lung disease remain unclear and controversial. We herein report the first case of DIP complicated with IgG4-related lung disease. Even if a patient has a smoking history, we emphasize the importance of exploring the association between DIP and IgG4-related lung disease to clarify the pathogenesis of these two disorders.
\end{abstract}

Key words: IgG4-related respiratory disease, desquamative interstitial pneumonia, autoimmune disorder

(Intern Med 56: 1553-1556, 2017)

(DOI: 10.2169/internalmedicine.56.8110)

\section{Introduction}

Desquamative interstitial pneumonia (DIP) is a rare form of interstitial lung disease first described in adults by Liebow et al. in 1965 (1). This disease has been linked to smoking frequency and carries a relatively favorable prognosis (2). In contrast, IgG4-related disease, which is a newly recognized fibro-inflammatory condition characterized by several features and previously referred to as IgG4-related sclerosing disease or hyper-IgG4 disease, may occur in the lung and involve the alveolar parenchyma, airways, and pleura (3). Various pulmonary manifestations of IgG4-related disease have been reported. Inoue et al. found that the radiologic features of IgG4-related lung disease could be classified into four types: solid nodular, round-shaped groundglass opacity, alveolar interstitial, and bronchovascular (4). However, if the IgG4-related lesion is present only in the lung, with no lesions in other organs, the reliability of the diagnosis of IgG4-related lung disease is controversial.

To our knowledge, the complication of IgG4-related lung disease with DIP has not been reported previously. We recently encountered a case of DIP with IgG4-positive plasma cell infiltration in the alveolar septa, interlobular septa, and pleura.

\section{Case Report}

The patient was a 65-year-old Japanese man in whom an abnormal shadow on chest X-ray had been noted in January 2012 during a routine medical examination. He was later referred to our hospital in September 2014 because of progression of the abnormal shadow. He had no symptoms but was a current smoker (1 pack/day for 46 years), and he had no obvious history of exposure to any dust or extrinsic allergens. Chest auscultation revealed slight bilateral inspiratory fine crackles in the lower zone. The physical examination did not reveal clubbed fingers, Raynaud's phenomenon, eruptions, swelling, or pain in any joints.

Computed tomography (CT) of the chest showed changes typical of emphysema in the upper lung and bilateral ground-glass opacities appearing with thin-walled cysts pre-

\footnotetext{
${ }^{1}$ Department of Respiratory Medicine, Kanagawa Cardiovascular and Respiratory Center, Japan, ${ }^{2}$ Department of Respiratory Medicine, Tokyo Jikei University Hospital, Japan, ${ }^{3}$ Department of Pathobiology, Yokohama City University Graduate School of Medicine, Japan and ${ }^{4}$ Department of Pathology, Japan Red Cross Medical Center, Japan

Received for publication August 8, 2016; Accepted for publication October 4, 2016

Correspondence to Dr. Hideaki Yamakawa, hide1144@jikei.ac.jp
} 


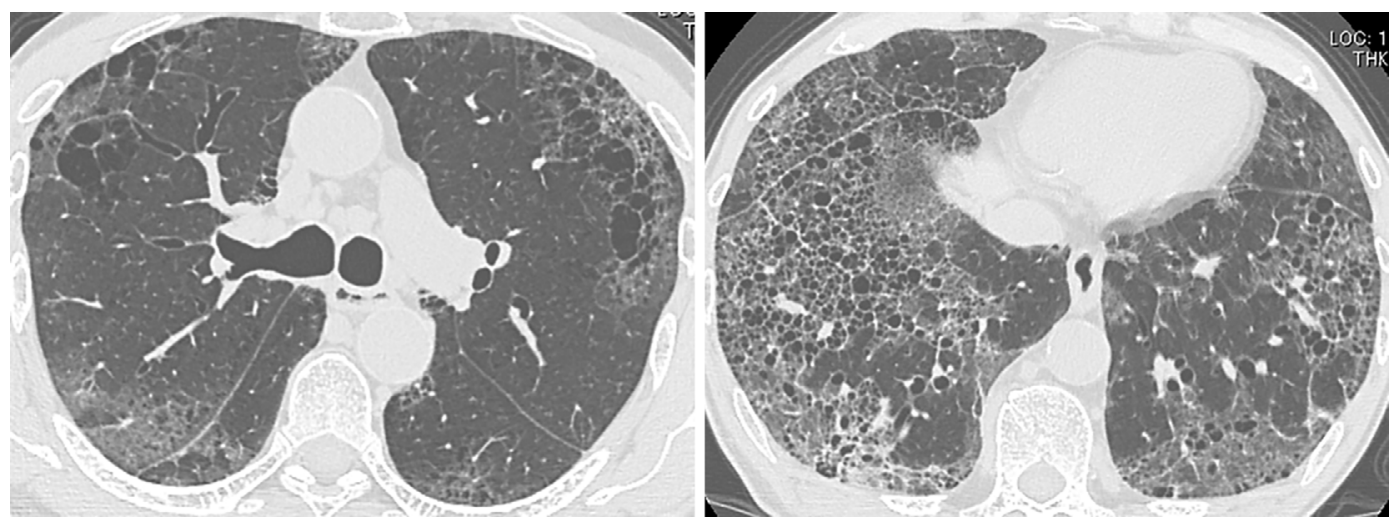

Figure 1. Chest computed tomography showed emphysema and bilateral ground-glass opacities with tiny, thin-wall cysts predominantly in the peripheral area of the lower lung. The tiny, thin-walled cysts formed within the regions of ground-glass opacities without honeycombing.

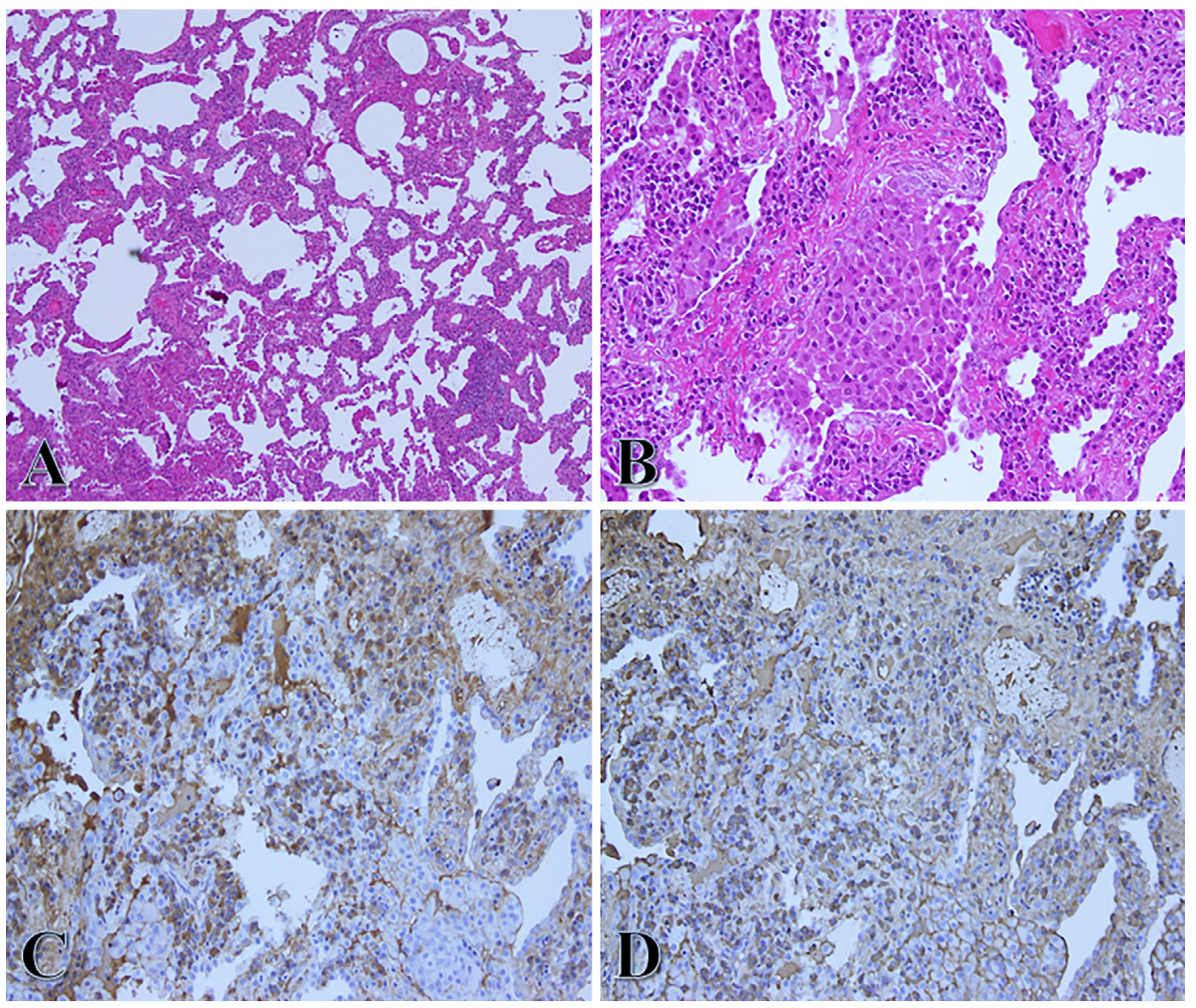

Figure 2. Histological images. (A) The lesion was characterized by an accumulation of large macrophages in the alveoli with inflammatory cell infiltration in the alveolar septa, pleura, and interlobular septa (Hematoxylin and Eosin (H\&E) staining, x100). (B) The lesion was an accumulation of pigmented eosinophilic macrophages in the alveolar spaces with infiltrating plasma cells and lymphocytes $($ H\&E staining, $\times 200)$. (C) IgG immunohistochemical staining revealed that most of the infiltrating plasma cells were positive $(\times 300)$. (D) IgG4 immunohistochemical staining revealed that approximately $75 \%$ of the IgG-positive plasma cells were positive for IgG4 $(\times 300)$.

dominantly in the peripheral area of the lower lung (Fig. 1). Laboratory examinations revealed significantly high serum levels of $\operatorname{IgG}(2,031 \mathrm{mg} / \mathrm{dL}), \operatorname{IgG} 4(907 \mathrm{mg} / \mathrm{dL})$, and $\mathrm{IgE}$ $(2,376 \mathrm{IU} / \mathrm{mL})$. The serum KL-6 and C-reactive protein lev- els were elevated to $756 \mathrm{U} / \mathrm{mL}$ and $0.45 \mathrm{mg} / \mathrm{dL}$, respectively. The test results for the autoantibodies examined were all negative. The bronchoalveolar lavage (BAL) fluid contained $5.9 \times 10^{5}$ cells $/ \mathrm{mL}, 85 \%$ macrophages, $6 \%$ lympho- 


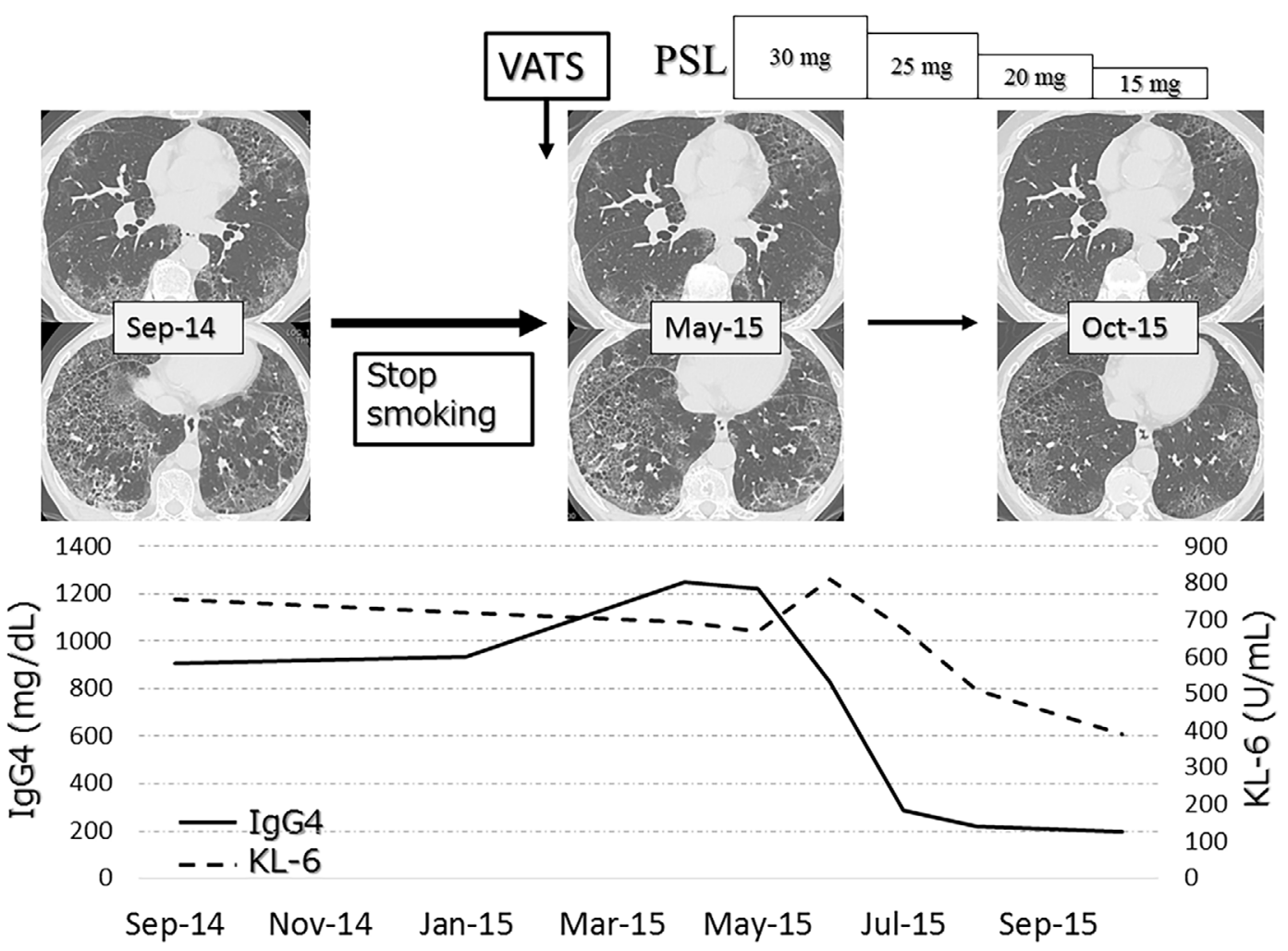

Figure 3. Clinical course. At six months after stopping smoking, the abnormal pulmonary shadow was slightly improved only in the left lower lobe, and the serum IgG4 and KL-6 levels were almost unchanged. Prednisolone (PSL) (30 mg/day) was started, and the dosage was gradually tapered, which led to an improvement in the radiological findings with normalization of the KL-6 and IgG4 levels. VATS: video-assisted thoracoscopic surgery

cytes, $1 \%$ neutrophils, and $8 \%$ eosinophils. The respiratory function tests showed evidence of a reduced diffusing capacity with a forced vital capacity (FVC) of $3.6 \mathrm{~L}(100.8 \%$, \% predicted), a forced expiratory volume in 1 second (FEV1) of $2.88 \mathrm{~L}(101.7 \%$, \% predicted), a FEV1/FVC ratio of $77.8 \%$, a diffusing capacity of carbon monoxide (DLCO) of $11.05 \mathrm{~mL} / \mathrm{min} /$ Torr $(76.3 \%$, \% predicted), and a DLCO/alveolar volume ratio of $62.2 \%$ (\% predicted). An ${ }^{18} \mathrm{~F}-$ fluorodeoxyglucose (FDG)-PET/CT scan showed slight FDG accumulation only in the pulmonary lesion and subcarinal lymph node. There were no other findings suggestive of IgG 4-related disease.

At 6 months after the patient had stopped smoking, there was almost no change in the abnormal pulmonary shadow and serum IgG4 and KL-6 levels. Thus, a video-assisted thoracoscopic biopsy of the lung was performed from the right S2 and S9 for diagnostic purposes. Histologically, the lesion was well defined, mainly by the interlobular septum, and showed an accumulation of large eosinophilic macrophages in the alveolar space with inflammatory cell infiltration in the alveolar septa, pleura, and interlobular septa (Fig. 2A, B). This lesion was histologically compatible with DIP. Furthermore, an immunohistochemical examination revealed that most of the infiltrating plasma cells were positive for $\mathrm{IgG}$ and $\mathrm{IgG} 4$. The ratio of $\mathrm{IgG}^{+}$to $\mathrm{IgG}^{+}$plasma cells was approximately 75\% (Fig. 2C, D).
Based on the clinical and histological findings, the patient was diagnosed as having IgG4-related respiratory disease with DIP, and treatment with prednisolone (PSL) $30 \mathrm{mg} /$ day $(0.5 \mathrm{mg} / \mathrm{kg} /$ day $)$ was started. The PSL dosage was gradually tapered, which led to an improvement in the radiological findings and normalization of the KL-6 and IgG4 levels (Fig. 3).

\section{Discussion}

We experienced a case of DIP complicated with IgG4related lung disease. DIP is thought to be a smoking-related interstitial pneumonia in the majority of cases (5). The radiological findings include widespread, patchy ground-glass opacifications with a predilection for the lower-lung zone and cyst formation. The main histological feature is the accumulation of numerous pigmented macrophages within most of the distal airspace of the lung (6). Our patient was diagnosed with DIP based on the presence of features compatible with this characterization.

The clinicopathological features of IgG4-related disease in the lung have been gradually clarified over time and usually manifest as inflammatory pseudotumors or interstitial pneumonia (7). Umehara et al. reported the comprehensive diagnostic criteria for IgG4-related disease as follows: a clinical examination showing characteristic diffuse/localized swelling 
or masses in single or multiple organs, elevated serum IgG4 concentrations ( $\geq 135 \mathrm{mg} / \mathrm{dL}$ ), and a histopathological examination showing marked lymphocyte and plasmacyte infiltration and fibrosis, with infiltration of $\mathrm{IgG} 4^{+}$plasma cells and a ratio of $\mathrm{IgG}^{+} / \mathrm{IgG}^{+}$cells of $>40 \%$ and $>10 \mathrm{IgG}^{+}$plasma cells/HPF (8). The features of our case met all of these criteria, and thus the diagnosis of IgG4-related lung disease was reasonable. To our knowledge, the present report presents the first case of IgG4-related lung disease with DIP.

A few cases of IgG4-related interstitial pneumonia have been newly reported $(4,9-12)$. Bilateral ground-glass opacities were the most common radiological findings, followed by reticular shadows $(4,12)$. Based on the most recent guidelines on idiopathic pulmonary fibrosis (13), most cases have a nonspecific interstitial pneumonia (NSIP) pattern and are inconsistent with the usual interstitial pneumonia (UIP) pattern due to the presence of wide-ranging ground-glass opacities, as in our case. Pathologically, the majority of cases have been NSIP, with only very rare cases of UIP and organizing pneumonia reported (9-12). Although storiform fibrosis and obliterative phlebitis as the major histopathological features of IgG4-related disease were not seen in our case, Ikeda et al. (12) and Deshpande et al. (14) reported that these two features may be inconspicuous or absent in cases of IgG4-related interstitial pneumonia. However, the complication of IgG4-related interstitial pneumonia with DIP has not been reported previously.

The pathogenesis of IgG4-related disease is assumed to be from autoimmune and allergic responses, as serum autoantibodies such as anti-lactoferrin, rheumatoid factor, allergic disorder, and elevated levels of serum $\operatorname{IgE}$ are often detected $(15,16)$. In contrast, DIP is sometimes present in nonsmokers and has been reported in patients with autoimmune disorders (e.g., connective tissue disease, rheumatoid arthritis) (17). Kawabata et al. reported that the association of organ-specific immunological diseases, elevated erythrocyte sedimentation rate and $\operatorname{IgG}$ and $\operatorname{IgE}$ levels, and high BAL eosinophilia in DIP suggests that the pathogenesis is immunologically mediated as an allergic response (6). We therefore hypothesized that the genesis of DIP in our case might have been associated not only with the patient's smoking habit, but also IgG4 immunity and/or an allergic response.

In conclusion, we herein described the first known case of DIP complicated with IgG4-related lung disease. Even if a patient has a smoking history, the cause of DIP may not be explained solely by smoking. Although the pathogenesis of these two disorders remains unknown, and they may have been only coincidentally associated in our patient, this is an important consideration, given the undetermined association of DIP and IgG4-related disease. Further studies are needed to clarify the details of these two disorders and their association.
The authors state that they have no Conflict of Interest (COI).

\section{References}

1. Liebow AA, Steer A, Billingsley JG. Desquamative interstitial pneumonia. Am J Med 39: 369-404, 1965.

2. Carrington $C B$, Gaensler EA, Coutu RE, FitzGerald MX, Gupta RG. Natural history and treated course of usual and desquamative interstitial pneumonia. N Engl J Med 298: 801-809, 1978.

3. Ryu JH, Sekiguchi H, Yi ES. Pulmonary manifestations of immunoglobulin G4-related sclerosing disease. Eur Respir J 39: 180186, 2012.

4. Inoue $\mathrm{D}$, Zen $\mathrm{Y}$, Abo $\mathrm{H}$, et al. Immunoglobulin G4-related lung disease: CT findings with pathologic correlations. Radiology 251: 260-270, 2009

5. Yousem SA, Colby TV, Gaensler EA. Respiratory bronchiolitisassociated interstitial lung disease and its relationship to desquamative interstitial pneumonia. Mayo Clin Proc 64: 1373-1380, 1989.

6. Kawabata Y, Takemura T, Hebisawa A, et al. Eosinophilia in bronchoalveolar lavage fluid and architectural destruction are features of desquamative interstitial pneumonia. Histopathology 52: 194202, 2008.

7. Zen Y, Inoue D, Kitao A, et al. IgG4-related lung and pleural disease: a clinicopathologic study of 21 cases. Am J Surg Pathol 33: 1886-1893, 2009.

8. Umehara H, Okazaki K, Masaki Y, et al. Comprehensive diagnostic criteria for IgG4-related disease (IgG4-RD), 2011. Mod Rheumatol 22: 21-30, 2012.

9. Tanaka K, Nagata K, Tomii K, Imai Y. A case of isolated IgG4related interstitial pneumonia: a new consideration for the cause of idiopathic nonspecific interstitial pneumonia. Chest 142: 228-230, 2012.

10. Wibmer T, Kropf-Sanchen C, Rüdiger S, et al. Isolated IgG4related interstitial lung disease: unusual histological and radiological features of a pathologically proven case. Multidiscip Respir Med 8: 22, 2013.

11. Onishi Y, Kawamura T, Kagami R, Nakahara Y, Yoshiro M. IgG4related lung disease with organizing pneumonia effectively treated with azathioprine. Intern Med 53: 2701-2704, 2014.

12. Ikeda S, Sekine A, Baba T, et al. Abundant IgG4-positive plasma cells in interstitial pneumonia without extrathoracic lesions of IgG4-related disease: is this finding specific to IgG4-related lung disease? Histopathology 2016 (Epub ahead of print).

13. Raghu G, Collard HR, Egan JJ, et al. An official ATS/ERS/JRS/ ALAT statement: idiopathic pulmonary fibrosis: evidence-based guidelines for diagnosis and management. Am J Respir Crit Care Med 183: 788-824, 2011.

14. Deshpande V, Zen Y, Chan JK, et al. Consensus statement on the pathology of IgG4-related disease. Mod Pathol 25: 1181-1192, 2012.

15. Okazaki K, Uchida K, Ohana M, et al. Autoimmune-related pancreatitis is associated with autoantibodies and a Th1/Th2-type cellular immune response. Gastroenterology 118: 573-581, 2000.

16. Masaki Y, Dong L, Kurose N, et al. Proposal for a new clinical entity, IgG4-positive multiorgan lymphoproliferative syndrome: analysis of 64 cases of IgG4-related disorders. Ann Rheum Dis 68: 1310-1315, 2009.

17. Godbert B, Wissler MP, Vignaud JM. Desquamative interstitial pneumonia: an analytic review with an emphasis on aetiology. Eur Respir Rev 22: 117-123, 2013.

The Internal Medicine is an Open Access article distributed under the Creative Commons Attribution-NonCommercial-NoDerivatives 4.0 International License. To view the details of this license, please visit (https://creativecommons.org/licenses/ by-nc-nd/4.0/).

(C) 2017 The Japanese Society of Internal Medicine

http://www.naika.or.jp/imonline/index.html 